\title{
Advanced Data Assimilation and Predictability Studies on High-Impact Weather and Climate
}

\author{
Zhaoxia Pu, ${ }^{1}$ Song-You Hong, ${ }^{2}$ Yaohui $\mathrm{Li}^{3}{ }^{3}$ and Hann-Ming Henry Juang ${ }^{4}$ \\ ${ }^{1}$ Department of Atmospheric Sciences, University of Utah, Salt Lake City, UT 84112, USA \\ ${ }^{2}$ Department of Atmospheric Sciences, Global Environment Laboratory, Yonsei University, Seoul 120-749, Republic of Korea \\ ${ }^{3}$ Institute of Arid Meteorology, Chinese Academy of Meteorological Sciences, Lanzhou 730020, China \\ ${ }^{4}$ Environmental Modeling Center, National Centers for Environmental Prediction, NOAA, Camp Springs, MD 20746, USA \\ Correspondence should be addressed to Zhaoxia Pu, zhaoxia.pu@utah.edu
}

Received 31 December 2010; Accepted 31 December 2010

Copyright (C) 2010 Zhaoxia Pu et al. This is an open access article distributed under the Creative Commons Attribution License, which permits unrestricted use, distribution, and reproduction in any medium, provided the original work is properly cited.

High-impact weather and climate systems refer to those events that have significant social, ecological, and economic impacts (such as tropical cyclones, winter storms, floods, droughts, etc.). Accurate forecasts of these systems more rely on our understanding of the systems and better representation of them in numerical models. Over the last decade, significant progress has been made in data assimilation, model development, and model diagnostics to enhance the predictability of these high-impact weather and climate systems. This special issue was motivated by rapid development in these areas. Total 13 papers in this volume address various issues in related topics.

Accurate initial conditions are one of key factors that influence the predictability of high-impact weather and climate systems. Modern data assimilation techniques combine observations and model information to form dynamically and physically consistent initial conditions for numerical models. A number of contributions addressed the new development in data assimilation. So S. Lakshmivarahan and J. M. Lewis presented a forward sensitivity approach to dynamic data assimilation. Duality between the method and the standard 4D-Var assimilation using adjoint equations has been proved. Q. Xu et al. developed a 3.5-dimensional variational method for Doppler radar data assimilation, which could be potentially beneficial to the predictability of severe weather systems.

Tropical cyclones are among the most destructive weather-related natural phenomena. They are responsible for some of the costliest and deadliest natural disasters in the world. A number of contributes addressed the tropical cyclone predictability problem from various angles. Y. Wang et al. presented a mesoscale data assimilation method for tropical cyclone initialization, which particularly improves the representation of tropical cyclone vortex and environment conditions in model initial conditions. H. R. Winterbottom and Q. Xiao's paper emphasized on an intercomparison of observations from four Global Position System (GPS) Radio Occultation (RO) and in situ observations within tropical cyclones, making a suggestion for better utilize these data in data assimilation. The paper by L. Zhang and $\mathrm{Z}$. Pu assessed the impact of wind profile measurements from the future satellite based Doppler wind lidar mission with observing system simulation experiments. A. Wada and N. Usui's study examined the impact of ocean pre-existing conditions on the predictability of a typhoon case using a couple-atmosphereocean model at high resolution. They found that ocean preexisting conditions remarkably affect typhoon rainbands.

While many efforts have been made in improving mesoscale regional model's ability in tropical cyclone predictability, an effort has also been made in the use of global model for tropical cyclone forecasts. M. F. Wehner et al. presented a set of high-resolution global atmospheric simulations with a general circulation model, focusing on the model's ability to represent tropical storms and their statistics. They found that the model produces storms of hurricane strength with realistic dynamic features and reasonable statistics. Some issues arose from global model simulations were also addressed.

Cold waves commonly occur in higher latitudes during winter season. They could cause serious economical loss 
and cold-related death. The contribution by D. H. Prasad et al. presented numerical simulation results to a severe cold wave event occurred during January 2006 over Europe. They found that the model is able to simulate the occurrence of the cold wave in 1 to 3 days range though the intensity is weaker than observations.

The ultimate predictability of high-impact weather systems relies on the improvement in computer models themselves, since model errors are the key factors that cause inaccurate forecasts. A number of contributions addressed the improvement or evaluation of the physical parameterization in a numerical model, specifically, a popular mesoscale community Weather Research and Forecasting (WRF) model. H. H. Shin et al. described the implementation of the orographic gravity wave drag process induced by subgrid-scale orography in the global version of the WRF model. The sensitivity of this new implementation to shortwave radiation was evaluated. S. Y. Hong et al. evaluated the WRF double-moment 6-class microphysics scheme for precipitating convection, making a step forward to improve the quantitative precipitation forecasting. In paper by J. Jin et al., four land surface schemes in the WRF model were evaluated for their sensitivities to accurately simulate temperature over the western United States. As a follow-up study of the WRF land surface schemes, J. Jin et al. also presented results to examine the impact of land change on the local climate over the Tibetan Plateau.

Although there have been many efforts made in improving initial conditions and computer models, due to inadequate observations, our limited understanding of the physical processes of the atmosphere, and the chaotic nature of atmospheric flow, uncertainties always exist in modern numerical weather and climate prediction. Recent developments in ensemble forecasting and ensemble-based data assimilation have proved that there are promising ways to beat the forecast uncertainties. A contribution by $\mathrm{H}$. Zhang and $\mathrm{Z}$. Pu gave a comprehensive overview of fundamental problems and recent progress associated with ensemble forecasting and ensemble-based data assimilation. The usefulness of these methods in improving high-impact weather forecasting was also discussed.

\section{Acknowledgments}

We, the guest editors of this special issue of Advances in Meteorology (AMET), are grateful to all the authors, reviewers, and AMET stuff. We hope that the papers in this issue will stimulate further development and confidence building for improving predictability of high impact weather and climate systems. 

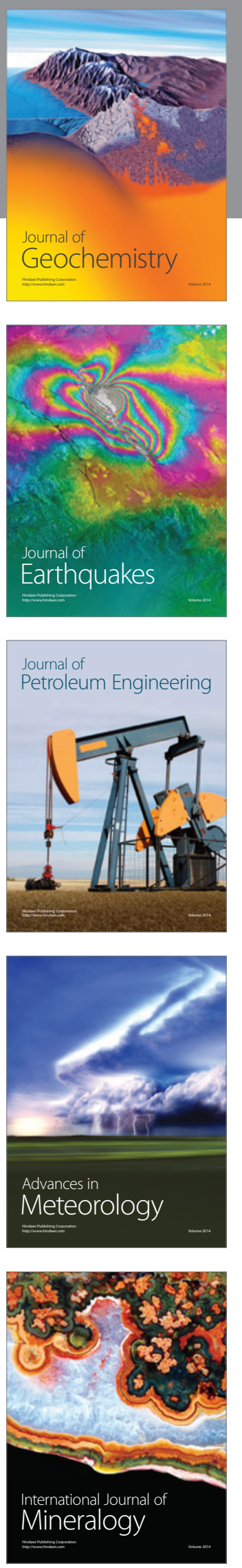
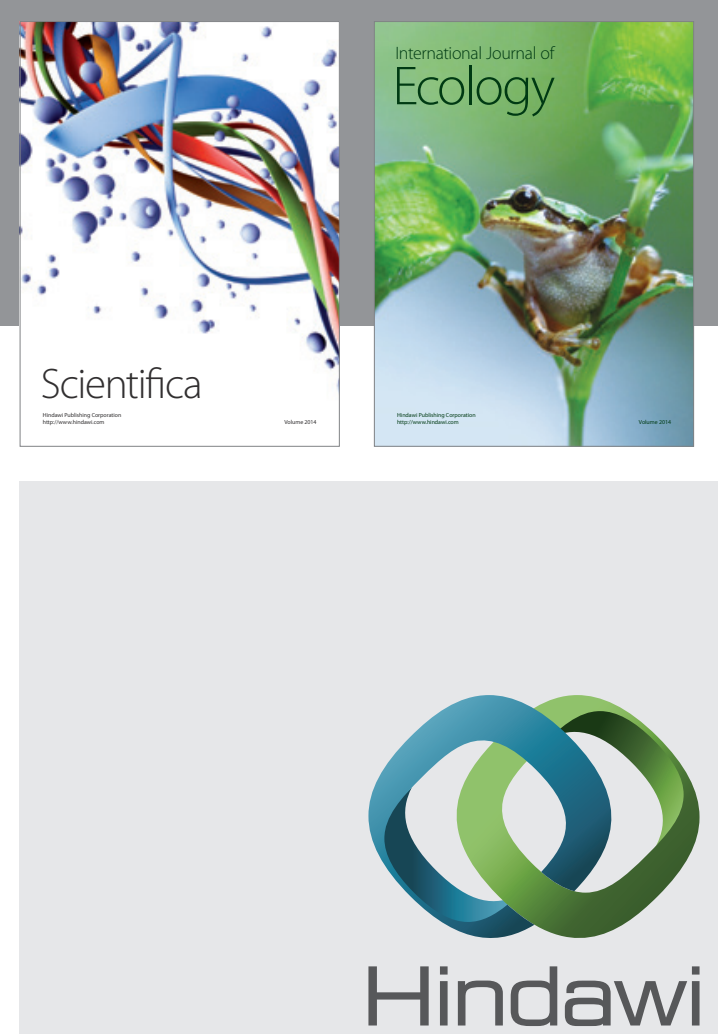

Submit your manuscripts at http://www.hindawi.com
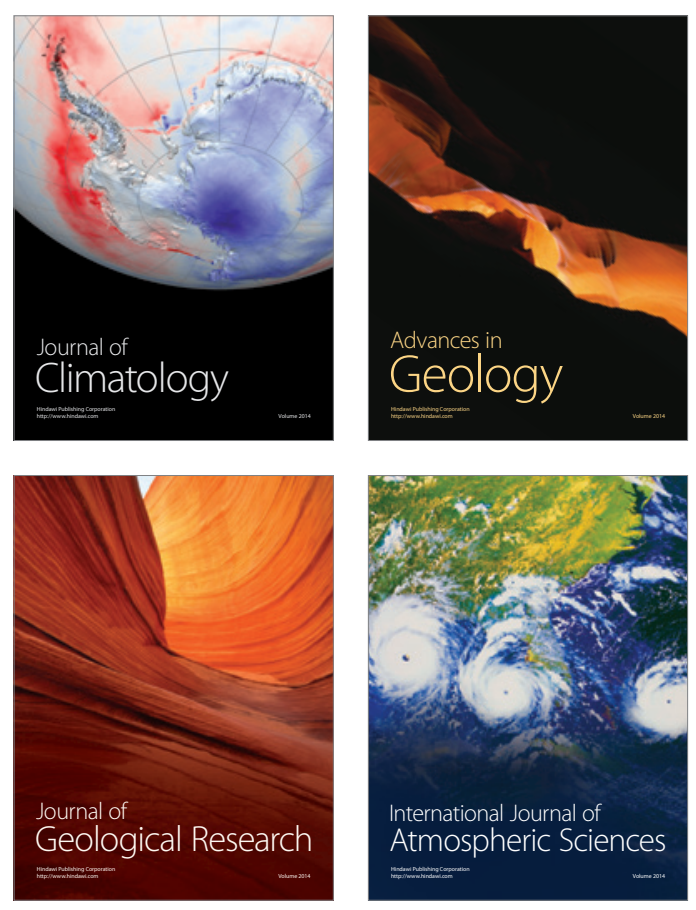
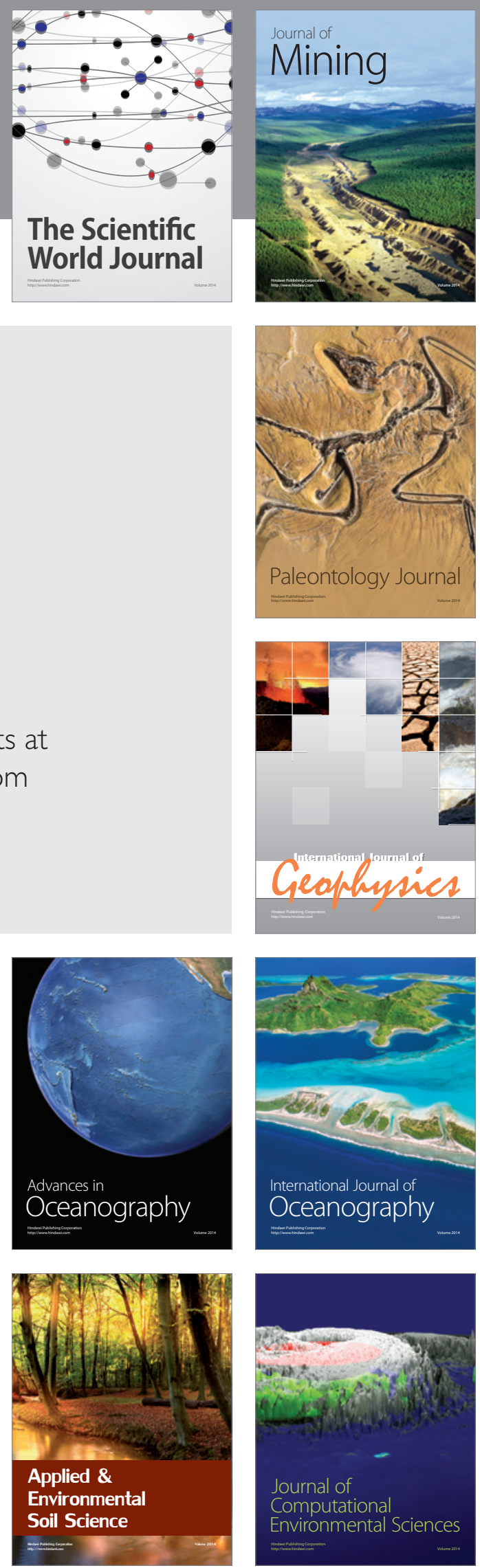